\title{
POSITION PAPER Oscillation Model for Describing Network Dynamics Caused by Asymmetric Node Interaction
}

\author{
Masaki AIDA $^{\dagger \mathrm{a})}$, Chisa TAKANO ${ }^{\dagger \dagger \mathrm{b})}$, Members, and Masayuki MURATA ${ }^{\dagger \dagger \mathrm{c}^{\circ}}$, Fellow
}

\begin{abstract}
SUMMARY This paper proposes an oscillation model for analyzing the dynamics of activity propagation across social media networks. In order to analyze such dynamics, we generally need to model asymmetric interactions between nodes. In matrix-based network models, asymmetric interaction is frequently modeled by a directed graph expressed as an asymmetric matrix. Unfortunately, the dynamics of an asymmetric matrix-based model is difficult to analyze. This paper, first of all, discusses a symmetric matrix-based model that can describe some types of link asymmetry, and then proposes an oscillation model on networks. Next, the proposed oscillation model is generalized to arbitrary link asymmetry. We describe the outlines of four important research topics derived from the proposed oscillation model. First, we show that the oscillation energy of each node gives a generalized notion of node centrality. Second, we introduce a framework that uses resonance to estimate the natural frequency of networks. Natural frequency is important information for recognizing network structure. Third, by generalizing the oscillation model on directed networks, we create a dynamical model that can describe flaming on social media networks. Finally, we show the fundamental equation of oscillation on networks, which provides an important breakthrough for generalizing the spectral graph theory applicable to directed graphs.

key words: spectral graph theory, coupled oscillators, node centrality, resonance, flaming, quantum theory
\end{abstract}

\section{Introduction}

Since the recent development and dissemination of information network technologies have activated information exchange on social networks, complex dynamics for describing propagation of activities on social media networks has become a rich source of research topics. In complex network analysis, there are many indices that can be used to describe the characteristics of networks, including degree distribution, clustering coefficient, and many kinds of node centralities [1]-[3]. Spectral graph theory is one of the key approaches for investigating the structure of networks and/or dynamics on networks, and the eigenvalues and the eigenvectors of the Laplacian matrix play important roles in investigating network structure and/or dynamics. Spec-

\footnotetext{
Manuscript received January 10, 2017.

Manuscript revised May 10, 2017.

Manuscript publicized July 3, 2017.

The author is with the Graduate School of Systems Design, Tokyo Metropolitan University, Hino-shi, 191-0065 Japan.

${ }^{\dagger}$ The author is with the Graduate School of Information Sciences, Hiroshima City University, Hiroshima-shi, 731-3194 Japan.

${ }^{+1 \dagger}$ The author is with the Graduate School of Information Science and Technology, Osaka University, Suita-shi, 565-0871
} Japan.

a)E-mail: aida@tmu.ac.jp

b) E-mail: takano@hiroshima-cu.ac.jp

c)E-mail: murata@ist.osaka-u.ac.jp

DOI: 10.1587/transcom.2017EBN0001 tral graph theory is applicable to many problems including clustering of networks, graph drawing, graph cut, node coloring, and image segmentation [4]-[6]. The Laplacian matrix is also important for describing the diffusion process on networks and the Markov process; the spectral graph theory has been applied to the consensus problem [7], [8]. One of the most significant properties of spectral graph theory is the fact that we can introduce graph Fourier transformation [9], [10], which is the diagonalization of the Laplacian matrix. The advantage of graph Fourier transformation can be found in its ability to decompose network dynamics into scales appropriate for the network's structure. As a result, complex network dynamics can be understood as the superposition of simple dynamics for each Fourier mode, and network dynamics can be completely understood algebraically.

However, the decomposition of dynamics into Fourier modes is effective only if the Laplacian matrix is symmetric. Moreover, almost all results of the spectral graph theory are effective only for symmetric Laplacian matrices. User dynamics on social or information networks is generated by the interaction between nodes on the networks. This interaction is generally asymmetric, that is, the strength of actions between nodes depends on link direction. To represent asymmetric actions on links, directed graphs are frequently used. Since the structure of a directed graph is normally expressed by an asymmetric matrix, graph Fourier transformation cannot be applied.

One proposal that uses spectral graph theory for directed graphs transforms asymmetric Laplacian matrixes in Jordan canonical form via elementary transformation [11], [12]. However, since asymmetric Laplacian matrices do not have the same convenient properties that the symmetric Laplacian matrices have, decomposition of the dynamics into simple Fourier modes remains unavailable.

This position paper proposes and introduces an oscillation model on networks in order to describe the dynamics of activity propagation on social media networks. In addition, we outline the following research topics derived from the proposed oscillation model.

First of all, we classify link asymmetry of networks into two types depending on whether it can be represented using node characteristics or not. For the first type, we show that the structure of a directed graph can be represented by a symmetric scaled Laplacian matrix. In addition, we analyze oscillation dynamics on networks to describe the propagation of activities on directed networks by using symmetric scaled Laplacian matrices. Next, we show the importance 
of the proposed oscillation model by explaining its relation to node centrality. The oscillation model described by the symmetric scaled Laplacian matrix can derive the generalized notion of node centrality, and it reduces to well-known indices of node centrality in simple cases. This makes it possible to associate the proposed oscillation model to actual dynamics on real networks. After that, as an application of oscillation model, we propose a framework to estimate eigenvalues of the scaled Laplacian matrix by observing the resonance of oscillation of the networks. This makes it possible to connect the theoretical link weight that describes the strength of interaction between nodes to observations of the dynamics demonstrated by real networks.

Second, we generalize the oscillation model to cover general link asymmetry. In the generalized oscillation model, the strength of node centrality derived from the oscillation model diverges under certain conditions. This phenomenon is generated from the network system itself and we propose that the phenomenon corresponds to the 'flaming' common in social media networks. We also introduce the direction of technology that may prevent or suppress flaming.

Finally, to describe the effect from asymmetric link causally, we propose the fundamental equation of network oscillation. The proposed equation has essentially the same form as the wave equation in relativistic quantum mechanics. This approach provides a significant breakthrough in generalizing the conventional spectral graph theory to general directed networks.

This paper is organized as follows. In Sect. 2, after defining the Laplacian matrix for directed networks, we classify the link asymmetry and introduce the scaled Laplacian matrix. In Sect. 3, we analyze oscillation models to describe the propagation of node activity on networks by using the symmetric scaled Laplacian matrix. In Sect. 4, we propose the oscillation energy of each node as an extended metric of node centrality and discuss the relationship to conventional node centralities. In Sect. 5, we propose the network resonance method to estimate eigenvalues of the scaled Laplacian matrix. In Sect. 6, we generalize the oscillation model to general asymmetric networks, and show a dynamical model for the flaming common in social media networks. In Sect. 7, we propose the fundamental equation of oscillation on general asymmetric networks In Sect.8, we show comprehensive discussion to compare our research with related work. Finally, we conclude this paper in Sect. 9.

\section{Scaled Laplacian Matrix for Describing Asymmetric Link Direction}

\subsection{Definition of the Laplacian Matrix}

Network structure is frequently expressed as a matrix. Let us consider loop-free directed graph $\mathcal{G}(V, E)$ with $n$ nodes, where $V=\{1,2, \ldots, n\}$ is the set of nodes and $E$ is the set of directed links. In addition, let the link weight for link $(i \rightarrow j) \in E$ be $w_{i j}>0$. We define the following $n \times n$ square

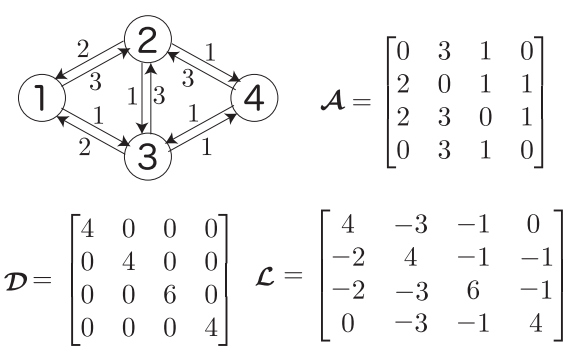

Fig. 1 An example of the Laplacian matrix.

matrix $\mathcal{A}=\left[\mathcal{A}_{i j}\right]$ as

$$
\mathcal{A}_{i j}:=\left\{\begin{array}{cc}
w_{i j} & ((i \rightarrow j) \in E), \\
0 & ((i \rightarrow j) \notin E) .
\end{array}\right.
$$

This matrix represents link presence and weights, and is called the (weighted) adjacency matrix.

Next, we define the weighted out-degree, $d_{i}$, of node $i$ $(i=1,2, \ldots, n)$ as

$$
d_{i}:=\sum_{j \in \partial i} w_{i j},
$$

where $\partial i$ denotes the set of nodes adjacent from node $i$. Also, weighted out-degree matrix $\mathcal{D}$ is defined as

$$
\mathcal{D}:=\operatorname{diag}\left(d_{1}, \ldots, d_{n}\right) \text {. }
$$

If all link weights are $w_{i j}=1$ for ${ }^{\vee}(i \rightarrow j) \in E, d_{i}$ is reduced to out-degree, i.e. the number of outgoing links from node $i$.

Based on the above preparation, we define the Laplacian matrix $\mathcal{L}$ of directed graph $\mathcal{G}(V, E)$ as follows [4], [5] (Fig. 1).

$$
\mathcal{L}:=\mathcal{D}-\mathcal{A} .
$$

The Laplacian matrix is also called the graph Laplacian. Note that since the row sum of the Laplacian matrix $\mathcal{L}$ is $0, \mathcal{L}$ has 0 as an eigenvalue. In addition, it is known that the multiplicity of the eigenvalue 0 is equal to the number of connected components of the corresponding graph. So, for a connected graph, the multiplicity is 1 . Hereafter, we consider a connected graph without loss of generality.

2.2 Symmetrization of Laplacian Matrix and the Scaled Laplacian Matrix

Although the Laplacian matrix $\mathcal{L}$ for a directed graph is generally an asymmetric matrix, we can classify the link asymmetry into two types: symmetrizable and unsymmetrizable. Figure 2 shows typical examples of link asymmetry. Figure 2(a) shows a hub type relation typical of the relation of a major blogger and the followers, and (b) shows a cyclic relation like rock-paper-scissors. Note that the link asymmetry in (a) can be expressed by node characteristic, while that in (b) is a pure link characteristic. That is, the hub node is strong and the other nodes are weak, in (a). This means that 


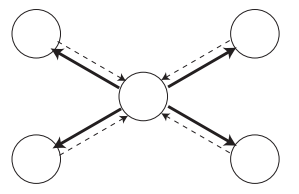

(a) hub type relation

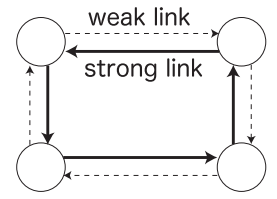

(b) cyclic relation
Fig. 2 Typical example of link asymmetry.

the link asymmetry in directed network like (a) can be expressed by using node characteristics in an undirected graph (i.e. symmetrizable). Let us consider the conditions yielding the asymmetric relation classified as this type.

The asymmetric Laplacian matrix $\mathcal{L}$ has the left eigenvector ${ }^{t} \boldsymbol{m}$ associated with the eigenvalue 0 , that is,

$$
{ }^{t} \boldsymbol{m} \mathcal{L}={ }^{t} \mathbf{0},
$$

where ${ }^{t} \mathbf{0}:=(0, \ldots, 0)$. Note that the multiplicity of the eigenvalue 0 is 1 for a connected graph. For each component $m_{i}>0$ of the left eigenvector ${ }^{t} \boldsymbol{m}=\left(m_{1}, \ldots, m_{n}\right)$, we assume the following condition:

$$
m_{i} w_{i j}=m_{j} w_{j i} \quad\left(\equiv k_{i j}\right) .
$$

The physical meaning of this condition will be discussed in Sect. 3.1. The oscillation model described in the next section satisfies this condition. Incidentally, the condition (5) is known as the detailed balance condition in the theory of Markov chains (see Sect. 8).

Hereafter, we call the Laplacian matrix that satisfies (5) symmetrizable and denote it by $\mathcal{L}_{0}$. The procedure used to represent $\mathcal{L}_{0}$ by a symmetric matrix is shown as follows. First, we consider an undirected graph and introduce its Laplacian matrix $\boldsymbol{L}$ as $\boldsymbol{L}:=\boldsymbol{D}-\boldsymbol{A}$, where $\boldsymbol{A}=\left[A_{i j}\right]$ is defined as

$$
A_{i j}:=\left\{\begin{array}{cc}
k_{i j} & ((i, j) \in E), \\
0 & ((i, j) \notin E),
\end{array}\right.
$$

and

$$
\boldsymbol{D}=\operatorname{diag}\left(\sum_{j=1}^{n} A_{1 j}, \sum_{j=1}^{n} A_{2 j}, \ldots, \sum_{j=1}^{n} A_{n j}\right) .
$$

Since $k_{i j}=k_{j i}$ from (5), $\boldsymbol{L}$ is a symmetric Laplacian matrix for a certain undirected graph. By using $\boldsymbol{L}$, the asymmetric (but symmetrizable) Laplacian matrix $\mathcal{L}_{0}$ is expressed as

$$
\mathcal{L}_{0}=\boldsymbol{M}^{-1} \boldsymbol{L}
$$

where $\boldsymbol{M}:=\operatorname{diag}\left(m_{1}, \ldots, m_{n}\right)$ means the scaling factors of nodes. That is, we define a directed graph as symmetrizable iff all its links satisfy (5). Figure 3 shows a simple example of the procedure that leads to (8).

Here, we introduce the scaled Laplacian matrix that is defined as

$$
\boldsymbol{S}_{0}:=\boldsymbol{M}^{-1 / 2} \boldsymbol{L} \boldsymbol{M}^{-1 / 2}=\boldsymbol{M}^{+1 / 2} \mathcal{L}_{0} \boldsymbol{M}^{-1 / 2} .
$$

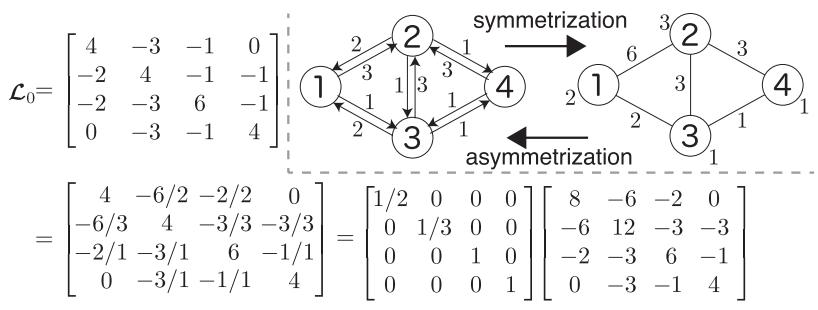

Fig. 3 An example of symmetrization of the Laplacian matrix for a directed graph.

Note that $\boldsymbol{S}_{0}$ is a symmetric matrix. Let $\boldsymbol{x}={ }^{t}\left(x_{1}, \ldots, x_{n}\right)$ be a (right) eigenvector associated with an eigenvalue $\lambda$ of $\mathcal{L}_{0}$, that is,

$$
\mathcal{L}_{0} x=\lambda \boldsymbol{x}
$$

By multiplying $\boldsymbol{M}^{+1 / 2}$ to this equation from the left, we obtain

$$
\boldsymbol{M}^{+1 / 2} \mathcal{L}_{0} \boldsymbol{x}=\boldsymbol{S}_{0}\left(\boldsymbol{M}^{+1 / 2} \boldsymbol{x}\right)=\lambda\left(\boldsymbol{M}^{+1 / 2} \boldsymbol{x}\right) .
$$

This means the scaled Laplacian matrix $\boldsymbol{S}_{0}$ has the same eigenvalues as $\mathcal{L}_{0}$, and its eigenvector is $\boldsymbol{y}:=\boldsymbol{M}^{+1 / 2} \boldsymbol{x}$. Since the quadratic form of $S_{0}$ is

$$
{ }^{t} \boldsymbol{y} \boldsymbol{S}_{0} \boldsymbol{y}=\sum_{(i, j) \in E} k_{i j}\left(\frac{y_{i}}{\sqrt{m_{i}}}-\frac{y_{j}}{\sqrt{m_{j}}}\right)^{2} \geq 0,
$$

the eigenvalues of $\boldsymbol{S}_{0}$ (also $\mathcal{L}_{0}$ ) are nonnegative. Let us consider sorting the eigenvalues in ascending order,

$$
0=\lambda_{0}<\lambda_{1} \leq \lambda_{2} \leq \cdots \leq \lambda_{n-1} .
$$

We can choose eigenvector $\boldsymbol{v}_{\mu}(\mu=0,1, \ldots, n-1)$ as the orthonormal eigenvector associated with $\lambda_{\mu}$. That is,

$$
\boldsymbol{S}_{0} \boldsymbol{v}_{\mu}=\lambda_{\mu} \boldsymbol{v}_{\mu}, \quad \boldsymbol{v}_{\mu} \cdot \boldsymbol{v}_{v}=\delta_{\mu v},
$$

where $\delta_{\mu v}$ denotes the Kronecker delta.

\section{Oscillation Models on Networks}

\subsection{Oscillation Model on Symmetrizable Directed Net- works}

To describe the propagation of the activity of a node across a network, let us consider oscillation dynamics on networks. The reason why we consider oscillation dynamics lies in the relationship between the oscillating phenomena and the well-known indices of node centrality. Details of the relationship are discussed in Sect. 4.

Let variable $x_{i}$ of node $i$ be displacement from equilibrium, and let its restoring force be proportional to the difference in the displacements of adjacent nodes. Figure 4 is a representative image of our oscillation model. Although the figure shows a 1-dimensional network, it is easily extended to general networks. To represent diverse oscillating behavior, we allow the spring constant of each link to be different 


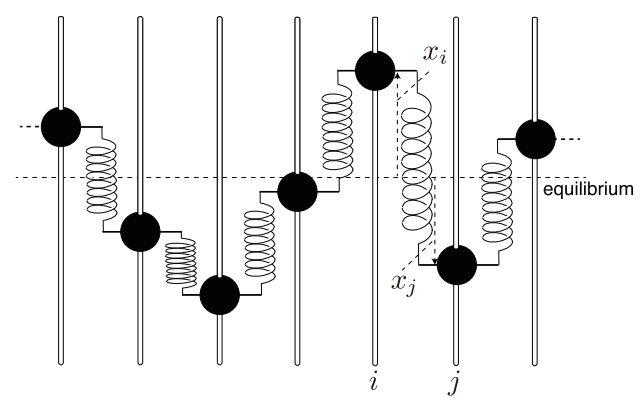

Fig. 4 Oscillation model on networks.

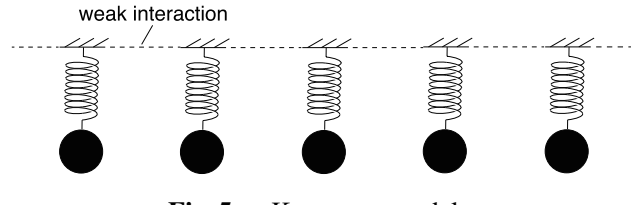

Fig. 5 Kuramoto model.

and the mass of each node to also be different.

Here, it is worthwhile discussing the validity of the oscillation model whose restoring force is proportional to the difference of displacements. Let the restoring force of node $i$ from node $j$ be a function $f(\Delta x)$ of the difference $\Delta x:=x_{i}-x_{j}$ of the displacements of adjacent nodes $i$ and $j$. It is natural to assume $f(\Delta x)=0$ if $\Delta x=0$. For small $\Delta x$, we can expand $f(\Delta x)$ as

$$
f(\Delta x)=-k_{i j} \Delta x+O\left(\Delta x^{2}\right),
$$

where $k_{i j}$ is a positive constant corresponding to the spring constant. So, our oscillation model can be considered as the basic and universal model if nonlinear effects in $O\left(\Delta x^{2}\right)$ are relatively small. A typical example that satisfies (14) is interaction between users on social media networks. Algorithmic trading in finance is likely to be an example of interaction between non-human objects. In future, an intelligent non-human node supported by artificial intelligence might participate as an interacting object.

Incidentally, the Kuramoto model is a well-known oscillation model on networks, see Fig. 5 [13]. Since this model consists of the same (or similar) oscillators coupled by weak interaction, and mainly describes the synchronization of these oscillators, it differs from our oscillation model.

We assign a spring constant to each link and express it as link weight $k_{i j}>0$. In addition, we assign mass $m_{i}>0$ to each node $i$. The equation of motion of the node displacement vector $\boldsymbol{x}(t):={ }^{t}\left(x_{1}(t), \ldots, x_{n}(t)\right)$ for the nondamped oscillation on networks is obtained as follows (see Appendix):

$$
\boldsymbol{M} \frac{\mathrm{d}^{2} \boldsymbol{x}(t)}{\mathrm{d} t^{2}}=-\boldsymbol{L} \boldsymbol{x}(t),
$$

or, by multiplying $\boldsymbol{M}^{-1}$ from the left,

$$
\frac{\mathrm{d}^{2} \boldsymbol{x}(t)}{\mathrm{d} t^{2}}=-\mathcal{L}_{0} \boldsymbol{x}(t),
$$

where $\boldsymbol{M}$ is the mass matrix $\boldsymbol{M}:=\operatorname{diag}\left(m_{1}, \ldots, m_{n}\right)$. Note that the equation of motion (15) reflects the asymmetric characteristics of links described by the asymmetric Laplacian matrix (8). Note that $\mathcal{L}_{0}$ is, in this case, a Laplacian matrix for the symmetrizable directed graph. The node characteristic $m_{i}$ in (5) corresponds to the mass of node $i$ in the oscillation model. In addition the condition (5) represents Newton's third law (the law of action and reaction). To symmetrize the equation of motion, we introduce vector $\boldsymbol{y}$ which is defined by

$$
\boldsymbol{y}(t)=\boldsymbol{M}^{+1 / 2} \boldsymbol{x}(t),
$$

where the equation of motion is written as

$$
\frac{\mathrm{d}^{2} \boldsymbol{y}(t)}{\mathrm{d} t^{2}}=-\boldsymbol{S}_{0} \boldsymbol{y}(t) .
$$

It follows that the equation of motion will yield the eigenvalue problem of the symmetric scaled Laplacian matrix.

Let $\boldsymbol{y}(t)$ be expanded by the eigenbasis $\boldsymbol{v}_{\mu}$ of $\boldsymbol{S}_{0}$ as

$$
\boldsymbol{y}(t)=\sum_{\mu=0}^{n-1} a_{\mu}(t) \boldsymbol{v}_{\mu},
$$

and solve the equation of motion for the Fourier mode $a_{\mu}(t)$ $(\mu=0,1, \ldots, n-1)$,

$$
\frac{\mathrm{d}^{2} a_{\mu}(t)}{\mathrm{d} t^{2}}=-\lambda_{\mu} a_{\mu}(t) .
$$

The procedure of expansion by eigenbasis is known as graph Fourier transformation [9], [10]. The solution is given by

$$
a_{\mu}(t)=a_{\mu}(0) e^{ \pm \mathrm{i} \omega_{\mu} t},
$$

where $\omega_{\mu}=\sqrt{\lambda_{\mu}}, \mathrm{i}=\sqrt{-1}$. The initial condition $a_{\mu}(0)=$ $\left|a_{\mu}(0)\right| e^{\mathrm{i} \theta_{\mu}}\left(-\pi<\theta_{\mu} \leq \pi\right)$ gives the amplitude $\left|a_{\mu}(0)\right|$ and phase $\theta_{\mu}$ of the corresponding Fourier mode, $\mu$.

The solution (18) means that the oscillation dynamics on symmetrizable directed networks can be expressed by using decomposition into the equation of motion of the harmonic oscillator (17) for each Fourier mode. The solution of oscillation on networks (the solution of (15)) is expressed as

$$
\boldsymbol{x}(t)=\boldsymbol{M}^{-1 / 2}\left(\sum_{\mu=0}^{n-1} a_{\mu}(0) e^{ \pm \mathrm{i} \omega_{\mu} t} \boldsymbol{v}_{\mu}\right) .
$$

Note that the appearance of oscillation behavior (19) varies widely with phase $\theta_{\mu}$ even if amplitude $\left|a_{\mu}(0)\right|$ is fixed. Consequently, to understand the universal aspect of oscillation dynamics, a kind of phase-free index is required. This issue is discussed in Sect. 4.

\subsection{Damped Oscillation Model}

In actual situations, any oscillation is damped with time. This subsection introduces a model for damped oscillation 
on networks.

Let us consider the equation of motion for damped oscillation

$$
\boldsymbol{M} \frac{\mathrm{d}^{2} \boldsymbol{x}(t)}{\mathrm{d} t^{2}}+\gamma \boldsymbol{M} \frac{\mathrm{d} \boldsymbol{x}(t)}{\mathrm{d} t}=-\boldsymbol{L} \boldsymbol{x}(t)
$$

where $\gamma$ is a constant. Here $\gamma \boldsymbol{M}$ means the viscous damping coefficient, where it is important to note that the viscous damping coefficient is assumed to be proportional to node mass. By using vector $\boldsymbol{y}(t)=\boldsymbol{M}^{+1 / 2} \boldsymbol{x}(t)$, we can symmetrize the equation of motion as

$$
\frac{\mathrm{d}^{2} \boldsymbol{y}(t)}{\mathrm{d} t^{2}}+\gamma \frac{\mathrm{d} \boldsymbol{y}(t)}{\mathrm{d} t}=-\boldsymbol{S}_{0} \boldsymbol{y}(t)
$$

The equation of motion for Fourier mode $a_{\mu}(t)$ is expressed as

$$
\frac{\mathrm{d}^{2} a_{\mu}(t)}{\mathrm{d} t^{2}}+\gamma \frac{\mathrm{d} a_{\mu}(t)}{\mathrm{d} t}+\lambda_{\mu} a_{\mu}(t)=0 .
$$

To analyze the solution of this equation, we assume the solution takes the form of $a_{\mu}(t) \propto e^{\alpha t}$. By substituting this into the equation of motion, we obtain the characteristic equation

$$
\alpha^{2}+\gamma \alpha+\lambda_{\mu}=0
$$

There are three different solutions to the equation of motion according to the solution of the characteristic equation, $\alpha=-(\gamma / 2) \pm \sqrt{(\gamma / 2)^{2}-\lambda_{\mu}}$. In the case of $(\gamma / 2)^{2}<\lambda_{\mu}$, the solution describes damped oscillations,

$$
a_{\mu}(t)=a_{\mu}(0) \exp \left[-\frac{\gamma}{2} t \pm \mathrm{i} \sqrt{\lambda_{\mu}-(\gamma / 2)^{2}} t\right] .
$$

In the case of $(\gamma / 2)^{2}=\lambda_{\mu}$, the solution describes critical damping,

$$
a_{\mu}(t)=\left(a_{\mu}(0)+c_{\mu} t\right) e^{-(\gamma / 2) t},
$$

where $c_{\mu}$ is a constant. Finally, in the case of $(\gamma / 2)^{2}>\lambda_{\mu}$, the solution describes overdamping. Let $\alpha_{+}$and $\alpha_{-}$(both values are negative) denote the solutions of the characteristic equation, the solution of the equation of motion is

$$
a_{\mu}(t)=c_{\mu}^{+} e^{\alpha_{+} t}+c_{\mu}^{-} e^{\alpha_{-} t}
$$

where $c_{\mu}^{+}$and $c_{\mu}^{-}$are constants.

\subsection{Forced Oscillation Model}

This subsection introduces a forced oscillation model on networks. Let us consider the situation that we impose forced oscillation with angular frequency $\omega$ on a certain node, $j$, as an external force. The equation of motion of the forced oscillation is

$$
\boldsymbol{M} \frac{\mathrm{d}^{2} \boldsymbol{x}(t)}{\mathrm{d} t^{2}}+\gamma \boldsymbol{M} \frac{\mathrm{d} \boldsymbol{x}(t)}{\mathrm{d} t}+\boldsymbol{L} \boldsymbol{x}(t)=(F \cos \omega t) \mathbf{1}_{\{j\}},
$$

where $F$ is a constant and $\mathbf{1}_{\{j\}}$ is only the $j$-th component that is 1 , all other components are 0 , that is,

$$
\mathbf{1}_{\{j\}}={ }^{t}(0, \ldots, 0, \stackrel{j}{\mathrm{~V}}, 0, \ldots, 0) .
$$

By using vector $\boldsymbol{y}=\boldsymbol{M}^{+1 / 2} \boldsymbol{x}$, the equation of motion can be symmetrized as

$$
\frac{\mathrm{d}^{2} \boldsymbol{y}(t)}{\mathrm{d} t^{2}}+\gamma \frac{\mathrm{d} \boldsymbol{y}(t)}{\mathrm{d} t}+\boldsymbol{S}_{0} \boldsymbol{y}(t)=\frac{F \cos \omega t}{\sqrt{m_{j}}} \mathbf{1}_{\{j\}} .
$$

Since $\boldsymbol{y}(t)$ depends on $\omega$, we redefine $\boldsymbol{y}(\omega, t):=\boldsymbol{y}(t)$. By expanding $\boldsymbol{y}(\omega, t)$ and $\mathbf{1}_{\{j\}}$ using the eigenbasis of the scaled Laplacian matrix $\boldsymbol{S}_{0}$, we introduce the Fourier modes $a_{\mu}(\omega, t)$ and $b_{\mu}$ as

$$
\boldsymbol{y}(\omega, t)=\sum_{\mu=0}^{n-1} a_{\mu}(\omega, t) \boldsymbol{v}_{\mu}, \quad \mathbf{1}_{\{j\}}=\sum_{\mu=0}^{n-1} b_{\mu} \boldsymbol{v}_{\mu} .
$$

The equation of motion of Fourier mode $a_{\mu}(\omega, t)$ is written as

$$
\frac{\partial^{2} a_{\mu}(\omega, t)}{\partial t^{2}}+\gamma \frac{\partial a_{\mu}(\omega, t)}{\partial t}+\lambda_{\mu} a_{\mu}(\omega, t)=\frac{F \cos \omega t}{\sqrt{m_{j}}} b_{\mu}
$$

The solution of the inhomogeneous equation (29) is the sum of the solutions of the corresponding homogeneous equation (21) and the particular solution of (29). Since the solution of the homogeneous equation (21) is dampened with time, only the oscillation of the particular solution of (29) remains after some long time. Since the angular frequency of the particular solution should be $\omega$, the particular solution can be expressed as

$$
\begin{aligned}
a_{\mu}(\omega, t) & =A_{\mu}(\omega) \cos \left(\omega t+\theta_{\mu}\right) \\
& =A_{\mu}(\omega)\left(\cos \omega t \cos \theta_{\mu}-\sin \omega t \sin \theta_{\mu}\right) .
\end{aligned}
$$

By substituting it into the equation of motion (29), the amplitude $A_{\mu}(\omega)$ and phase $\theta_{\mu}$ of the particular solution are given by

$$
\begin{aligned}
A_{\mu}(\omega) & =\frac{F b_{\mu}}{\sqrt{m_{j}}} \frac{1}{\sqrt{\left(\omega_{\mu}^{2}-\omega^{2}\right)^{2}+(\gamma \omega)^{2}}}, \\
\tan \theta_{\mu} & =-\frac{\gamma \omega}{\omega_{\mu}^{2}-\omega^{2}} .
\end{aligned}
$$

\section{Node Centrality and Oscillation Energy}

As shown in Sect. 3.1, the equation of motion (the wave equation) (15) cannot describe the phase of oscillations. Since the appearance of the behavior of the oscillating phenomenon strongly depends on the phase, it is hard to extract useful information from direct observation of oscillating aspects. Of course, since $a_{\mu}(t)$ of (18) is a complex-valued function, the value of $a_{\mu}(t)$ cannot be observed in actual networks. This section introduces the oscillation energy of each node as a non-negative-valued phase-free index, and shows that it can reproduce the well-known indices of node centrality. This means that our oscillation model can give a 


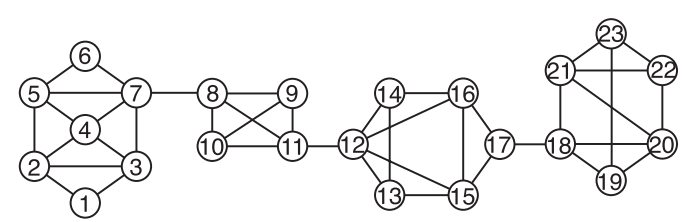

Fig. 6 Network model.
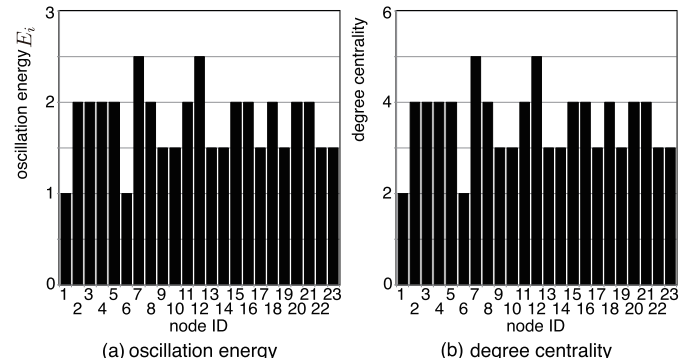

Fig. 7 Comparison between oscillation energy of each node and degree centrality.

generalization of node centrality.

For the oscillation model described in Sect. 3.1, we define node activity as the oscillation energy of the node. From (18), oscillation behavior is decomposed into $n$ independent harmonic oscillators. Since the oscillation energy of a harmonic oscillator with mass $m$, natural frequency $\omega$, and amplitude $A$ is given by $\frac{1}{2} m \omega^{2} A^{2}$, the oscillation energy $E_{i}$ of node $i$ is obtained by summing the oscillation energy for each Fourier mode, as

$$
\begin{aligned}
E_{i} & =\frac{1}{2} m_{i} \sum_{\mu=0}^{n-1}\left|\omega_{\mu} a_{\mu}(t) \frac{v_{\mu}(i)}{\sqrt{m_{i}}}\right|^{2} \\
& =\frac{1}{2} \sum_{\mu=0}^{n-1}\left|\omega_{\mu} a_{\mu}(t) v_{\mu}(i)\right|^{2},
\end{aligned}
$$

where $v_{\mu}(i)$ denotes the $i$-th component of the eigenbasis $\boldsymbol{v}_{\mu}$ associated with the eigenvalue $\lambda_{\mu}$ of the scaled Laplacian matrix $\boldsymbol{S}_{0}$, that is,

$$
\boldsymbol{v}_{\mu}=\left(v_{\mu}(1), \ldots, v_{\mu}(n)\right) .
$$

The oscillation energy of each node gives a generalization of node centrality [16], [17]. To demonstrate the relationship, we use the network model shown in Fig. 6, where all link weights and node masses are set at 1 . As the initial condition of the wave equation (15), we set the displacement of only one node. We call this node the source node of activity. Let us consider the situation that the source node of activity is chosen at random. In this case, all Fourier modes contribute at the same strength, so we set $\left|a_{\mu}(0)\right|=1$ for all $\mu$. Figure 7(a) shows the oscillation energy of each node and (b) shows the degree centrality (the number of links for each node). This comparison shows the oscillation energy of each node is proportional to degree centrality.

Next, let us consider the betweenness centrality, which is another well-known index of node centrality. Let the number of shortest paths between node $j$ and node $k$ be $\sigma_{j k}$,

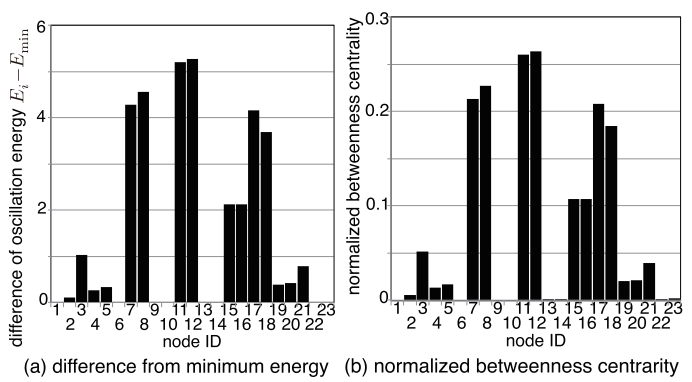

Fig. 8 Comparison between the difference in oscillation energy and the betweenness centrality.

and the number of those paths passing through the node $i$ be $\sigma_{j k}(i)$. The betweenness centrality $g(i)$ for node $i$ is defined as

$$
g(i):=\sum_{j, k \in \backslash\{i\}} \frac{\sigma_{j k}(i)}{\sigma_{j k}} .
$$

The normalized betweenness centrality $\bar{g}(i)$ is defined as

$$
\bar{g}(i):=\frac{2 g(i)}{(n-1)(n-2)} .
$$

The physical meaning of $\bar{g}(i)$ is the ratio of the number of shortest paths associated with node $i$ to the number of combination of node pairs in $V \backslash\{i\}$, that is $(n-1)(n-2) / 2$.

Let the link weight $w_{i j}\left(=w_{j i}\right)$ of the network model shown in Fig. 6 be set as the number of the shortest paths passing through link $(i, j)$. Figure 8(a) shows the difference between the oscillation energy for each node, $E_{i}$, and the minimum energy $E_{\min }$ defined as

$$
E_{\min }:=\min _{i \in V} E_{i} .
$$

Figure $8(\mathrm{~b})$ shows the normalized betweenness centrality $\bar{g}(i)$ for each node. We can recognize that the difference of the oscillation energ $E_{i}-E_{\min }$ is proportional to the betweenness centrality.

The relationships between the oscillation energy of each node and these node centralities can be proved mathematically [16], [17]. Assigning link weights and node masses flexibly yields a generalized node centrality. The oscillation energy can be defined even for damped oscillation on networks. Detailed discussion is presented in [16], [17].

\section{Network Resonance Method for Investigating the Eigenvalues of Network Dynamics}

Since the actual structure of social networks is difficult to know, it is almost impossible to measure components of the scaled Laplacian matrix $\boldsymbol{S}_{0}$, directly. For example, in social networks, the strength and/or significance of friendships (links) between two users is, in general, not proportional to the volume of traffic they exchange, and it is hard to observe. Thus the eigenvalues of $\boldsymbol{S}_{0}$, which is the key to describing the oscillation dynamics on networks, cannot be calculated from $\boldsymbol{S}_{0}$. However, since the oscillation energy is related to 


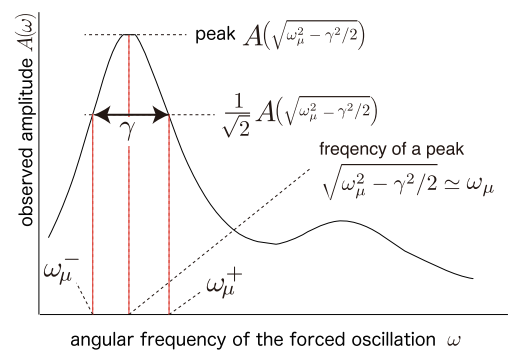

Fig. 9 Concept of network resonance.

the node centrality that is the strength of activity of node on networks, we expect that the observed node centrality can give a clue of the underlying structure of oscillation dynamics. The oscillation energy is related to the natural frequency and the amplitude of the oscillation. In this section, by applying the forced oscillation on networks, we examine how to estimate natural frequency (square root of eigenvalue) of $\boldsymbol{S}_{0}$ from observations of the amplitude that is obtained from observation of the oscillation energy.

As recognized from the discussion in Sect. 3.3, amplitude $A_{\mu}(\omega)$ of (31) takes maximal value at

$$
\omega=\sqrt{\omega_{\mu}^{2}-\gamma^{2} / 2}
$$

This phenomenon is called resonance. When we observe the oscillation of a node under forced oscillation, the mixture of oscillation (30) for each $\mu$, that is, $\boldsymbol{y}(\omega, t)$ of the first equation of (28) is observed. We propose a method to estimate eigenvalue $\lambda_{\mu}$ (or $\omega_{\mu}=\sqrt{\lambda_{\mu}}$ ) and damping factor $\gamma$ from observations of the amplitude

$$
A(\omega):=|\boldsymbol{y}(\omega, t)|
$$

of the response oscillation (Fig. 9). In practice, amplitude $A(\omega)$ is indirectly obtained from observations of oscillation energy.

The Q-factor represents the sharpness of amplitude $A_{\mu}(\omega)$ with respect to $\omega$. On both sides of the peak of amplitude $A_{\mu}(\omega)$, we define frequencies $\omega_{\mu}^{+}$and $\omega_{\mu}^{-}$that give the amplitudes $A_{\mu}\left(\omega_{\mu}^{+}\right)$and $A_{\mu}\left(\omega_{\mu}^{-}\right)$that are $1 / \sqrt{2}$ times the peak value of $A_{\mu}(\omega)\left(\omega_{\mu}^{+}>\omega_{\mu}^{-}\right)$. Since oscillation energy is proportional to the square of the amplitude, $\omega_{\mu}^{+}-\omega_{\mu}^{-}$means the half width of energy. The Q-factor is defined as

$$
Q_{\mu}:=\frac{\sqrt{\omega_{\mu}^{2}-\gamma^{2} / 2}}{\omega_{\mu}^{+}-\omega_{\mu}^{-}} .
$$

We assume $\gamma \ll \omega_{\mu}$ and approximate $A_{\mu}(\omega)$ around $\omega=\omega_{\mu}$. By using $\omega^{2}-\omega_{\mu}^{2} \simeq 2 \omega_{\mu}\left(\omega-\omega_{\mu}\right)$,

$$
\begin{aligned}
A_{\mu}(\omega) & \simeq \frac{F b_{\mu}}{\sqrt{m_{j}}} \frac{1}{\sqrt{\left(2 \omega_{\mu}\left(\omega-\omega_{\mu}\right)\right)^{2}+\left(\gamma \omega_{\mu}\right)^{2}}} \\
& =\frac{F b_{\mu}}{\sqrt{m_{j}} \omega_{\mu}} \frac{1}{\sqrt{4\left(\omega-\omega_{\mu}\right)^{2}+\gamma^{2}}} .
\end{aligned}
$$

Therefore,

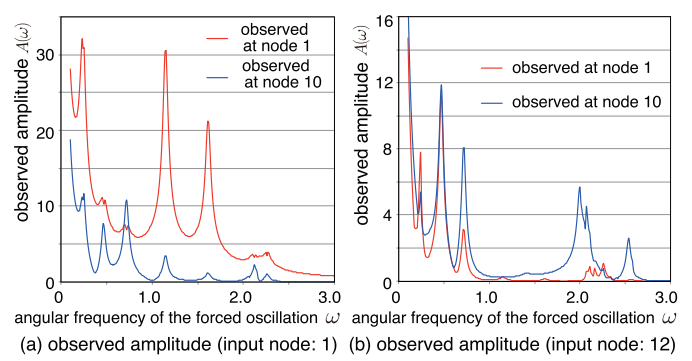

Fig. 10 Examples of network resonance: the amplitude of oscillation as a response to input oscillation with $\omega$.

$$
A_{\mu}\left(\omega_{\mu}\right) \simeq \frac{F b_{\mu}}{\sqrt{m_{j}} \omega_{\mu} \gamma}, \quad A_{\mu}\left(\omega_{\mu} \pm \gamma / 2\right) \simeq \frac{1}{\sqrt{2}} A_{\mu}\left(\omega_{\mu}\right),
$$

and we have $\omega_{\mu}^{ \pm}=\omega_{\mu} \pm \gamma / 2$ (double-sign indicates correspondence). Consequently, we have

$$
Q_{\mu} \simeq \frac{\omega_{\mu}}{\gamma}
$$

These relations enable us to estimate natural frequency $\omega_{\mu}$ (or the eigenvalue $\lambda_{\mu}=\omega_{\mu}^{2}$ ) and damping factor $\gamma$.

We use the network model shown in Fig. 6, i.e. all link weights and node masses equal 1. Figures 10(a) and (b) show examples of network resonance for external force inputs by node 1 and 12, respectively: the amplitude $A(\omega)=$ $|\boldsymbol{y}(\omega, t)|$ is observed at node 1 (red line) and node 10 (blue line) as the response of the external force with angular frequency $\omega$. Amplitude $A(\omega)$ exhibits a different aspect depending on which pair of input and observed nodes are selected. Therefore, we expect that eigenvalues of the scaled Laplacian matrix can be estimated from appropriate pairs of input and observed nodes.

Figure 11 compares the actual values of natural frequencies $\omega_{1}, \omega_{2}, \ldots, \omega_{6}$ and their estimated values obtained from Fig. 10(a) and (b). For example, "Estimation 01-10" in Fig. 11 means the input node is 1 and the observed node is 10. The estimated natural frequencies are close to the actual values. Depending on the positions of both the input node of forced oscillation and the observed node, there are natural frequencies that cannot be observed. For example, the values of $\omega_{2}, \omega_{3}, \omega_{4}$ and $\omega_{5}$ cannot be estimated from the node pair of input node 1 and observed node 1 . Selecting the appropriate pair of input and observed nodes avoids this problem.

The above estimation method is called the network resonance method. The network resonance method can estimate all the eigenvalues of the scaled Laplacian matrix $\boldsymbol{S}_{0}$ with high accuracy [18]. In addition, by analyzing peak height at resonance, it is expected that the absolute value of a component of eigenvector can be estimated by the network resonance method [19]. If we have all the eigenvalues and eigenvectors of $\boldsymbol{S}_{0}$, we can reproduce $\boldsymbol{S}_{0}$ algebraically. Therefore, the network resonance method can associate the Laplacian matrix that describes interaction between nodes and the observation of actual network dynamics, and provide viable explanations of the abstract interaction between 


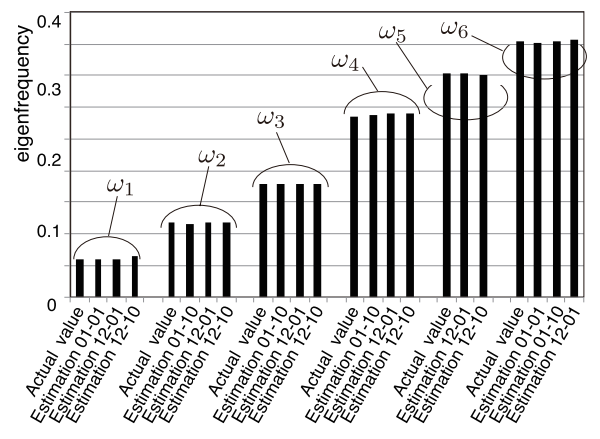

Fig. 11 Estimation of the natural frequency $\omega_{\mu} \mathrm{s}$.

nodes. Thus, the network resonance method suggests a way of estimating the significance of friendship.

\section{Dynamical Model of Flaming on Social Media Net- works}

In Sect. 3-Sect. 5, we focused on just symmetrizable graphs. Hereafter, we consider unsymmetrizable graphs (general directed graphs) and generalize the oscillation model to unsymmetrizable graphs. In addition, we discuss a dynamical model of flaming on social media networks, where we define that the flaming is the phenomenon that the node activity (oscillation energy) diverges with time.

Although Laplacian matrix $\mathcal{L}$ of a general directed graph $\mathcal{G}(V, E)$ is unsymmetrizable in general and (5) is not always satisfied, $\mathcal{L}$ can be decomposed into two parts as

$$
\mathcal{L}=\mathcal{L}_{0}+\mathcal{L}_{\mathrm{I}},
$$

where $\mathcal{L}_{0}$ is the Laplacian matrix for a symmetrizable network, and $\mathcal{L}_{\mathrm{I}}$ is a Laplacian matrix for the network having at most only one-way directed link between nodes. Note that the decomposition (36) is not unique, but we can choose appropriate decomposition for easy to analyze. The flexibility of this decomposition is advantageous for controlling and modeling network dynamics. Details are discussed at the end of this section, and Sects. 7-8.

Next, similar to the discussion in Sect. 2.2, we define the scaled Laplacian matrix $\boldsymbol{S}$ for general directed graphs as follows. Same as Sect. 2.2, let us consider the mass matrix $\boldsymbol{M}_{0}:=\operatorname{diag}\left(m_{1}, \ldots, m_{n}\right)$, which is a diagonal matrix and whose diagonal components are components of the left eigenvector $\boldsymbol{m}:={ }^{t}\left(m_{1}, \ldots, m_{n}\right)$ associated with the eigenvalue 0 of the symmetrizable Laplacian matrix $\mathcal{L}_{0}$,

$$
{ }^{t} \boldsymbol{m} \mathcal{L}_{0}={ }^{t} \mathbf{0} .
$$

Then the scaled Laplacian matrix $\boldsymbol{S}$ is defined as

$$
\boldsymbol{S}:=\boldsymbol{M}_{0}^{+1 / 2} \mathcal{L} \boldsymbol{M}_{0}^{-1 / 2} \text {. }
$$

By defining the scaled Laplacian matrix for symmetrizable portion $\mathcal{L}_{0}$ of the Laplacian matrix $\mathcal{L}$ as $\boldsymbol{S}_{0}$ := $\boldsymbol{M}_{0}^{+1 / 2} \mathcal{L}_{0} \boldsymbol{M}_{0}^{-1 / 2}$, we have a decomposition of the scaled Laplacian matrix $S$ as

$$
S=S_{0}+S_{\mathrm{I}},
$$

where $\boldsymbol{S}_{0}$ is a symmetric matrix. The decomposition (39) depends on the decomposition (36).

Next, we extend the oscillation model described in Sect. 3.1 to a general directed graph. Let $x_{i}(t)$ be the displacement of node $i$ at time $t$ on a directed graph $\mathcal{G}(V, E)$, and the restoring force that the node $i$ receives from the adjacent node $j$ be $-w_{i j}\left(x_{i}(t)-x_{j}(t)\right)$ [20]. Note that since $w_{i j} \neq w_{j i}$, the strength of restoring forces between nodes $i-j$ are asymmetric, in general.

Then, the equation of motion of the displacement vector $\boldsymbol{x}(t)={ }^{t}\left(x_{1}(t), \ldots, x_{n}(t)\right)$ can be written as

$$
\frac{\mathrm{d}^{2} \boldsymbol{x}(t)}{\mathrm{d} t^{2}}=-\mathcal{L} \boldsymbol{x}(t)=-\left(\mathcal{L}_{0}+\mathcal{L}_{\mathrm{I}}\right) \boldsymbol{x}(t),
$$

and the equation of motion of the vector $\boldsymbol{y}(t)=\boldsymbol{M}_{0}^{+1 / 2} \boldsymbol{x}(t)$ is obtained as

$$
\frac{\mathrm{d}^{2} \boldsymbol{y}(t)}{\mathrm{d} t^{2}}=-\boldsymbol{S} \boldsymbol{y}(t)=-\left(\boldsymbol{S}_{0}+\boldsymbol{S}_{\mathrm{I}}\right) \boldsymbol{y}(t) .
$$

Here, it is worthwhile to note that if the oscillation occurs on an unsymmetrizable graph i.e. $\mathcal{L}_{\mathrm{I}} \neq \mathbf{O}$ (O) is the null matrix), Newton's third law does not hold. This means that the generalized oscillation model cannot be depicted by a dynamical model like Fig. 4. In other words, the generalized oscillation model does not corresponds to dynamical phenomena in the real world, but to a kind of virtual phenomena in cyber space.

Following the discussion in Sect.3.2, the equation of motion of damped oscillation can be written as

$$
\frac{\mathrm{d}^{2} \boldsymbol{y}(t)}{\mathrm{d} t^{2}}-\gamma \frac{\mathrm{d} \boldsymbol{y}(t)}{\mathrm{d} t}=-\boldsymbol{S} \boldsymbol{y}(t)=-\left(\boldsymbol{S}_{0}+\boldsymbol{S}_{\mathrm{I}}\right) \boldsymbol{y}(t),
$$

where $\gamma \geq 0$ (it includes (41) for $\gamma=0$ ).

Since $S$ is no longer a real symmetric matrix, we should consider the following situations when solving the equation of motion (42):

- $S$ is not always diagonalizable.

- Eigenvectors of $\boldsymbol{S}$ cannot always be orthogonalized.

- Eigenvalues of $S$ are, in general, complex numbers.

Let us consider the impact of these situations on the solution of equation of motion (42) and the oscillation energy.

Let the eigenvalues of $\boldsymbol{S}$ be $\bar{\lambda}_{\mu}(\mu=0, \ldots, n-1)$ and the eigenvector associated with $\bar{\lambda}_{\mu}$ be $\overline{\boldsymbol{v}}_{\mu}$. The necessary condition that $\boldsymbol{S}$ is not diagonalizable is that $\boldsymbol{S}$ has multiple eigenvalues. In other words, the characteristic equation

$$
\operatorname{det}(\boldsymbol{S}-\bar{\lambda} \boldsymbol{I})=0,
$$

has repeated roots, where $\boldsymbol{I}$ is the unit matrix. In engineering, the value of link weights of actual networks is determined to the accuracy level of the significant digits expected. Therefore, the mathematical condition for repeated roots of (43) is easily avoided by changing the link weights only very slightly. This allows us to assume that $S$ has $n$ 
distinct eigenvalues.

The fact that the eigenvectors are not orthogonal means that the total oscillation energy is not obtained as the simple summation of oscillation energy of each oscillation mode, and thus that different oscillation modes are coupled. The coupling of different oscillation modes is discussed in the next section. In this section, we consider the impact of the fact that the eigenvalue is a complex number.

The eigenvectors associated with the different eigenvalues are generally not orthogonal, but it is guaranteed that they are linearly independent. Therefore, by using the following $n \times n$ square matrices

$$
\overline{\boldsymbol{\Lambda}}:=\operatorname{diag}\left(\bar{\lambda}_{0}, \ldots, \bar{\lambda}_{n-1}\right), \text { and } \overline{\boldsymbol{P}}:=\left(\overline{\boldsymbol{v}}_{0}, \ldots, \overline{\boldsymbol{v}}_{n-1}\right),
$$

$\boldsymbol{S}$ can be diagonalized as follows:

$$
\bar{\Lambda}=\overline{\boldsymbol{P}}^{-1} \boldsymbol{S} \overline{\boldsymbol{P}}
$$

where the existence of $\overline{\boldsymbol{P}}^{-1}$ arises from the linear independence of $\overline{\boldsymbol{v}}_{\mu}$. Incidentally, the eigenvalues of $\boldsymbol{S}_{0}$ are nonnegative and all the components of the eigenvectors have real values. However, the eigenvalues of $\boldsymbol{S}$ are generally complex numbers, and the components of the eigenvectors are also generally complex numbers.

Let us expand the solution $\boldsymbol{y}(t)$ of (42) by the eigenvectors of $\boldsymbol{S}$, as

$$
\boldsymbol{y}(t)=\sum_{\mu=0}^{n-1} \bar{a}_{\mu}(t) \overline{\boldsymbol{v}}_{\mu} .
$$

By substituting this into the equation of motion (42), and applying a similar procedure to that described in Sect. 3.2, the characteristic function for Ansatz $\bar{a}_{\mu}(t) \propto e^{\bar{\alpha} t}$ is obtained as

$$
\bar{\alpha}^{2}+\gamma \bar{\alpha}+\bar{\lambda}_{\mu}=0 .
$$

Since $\bar{\lambda}_{\mu}$ is a complex number, we define

$$
r e^{\mathrm{i} \theta}:=\bar{\lambda}_{\mu}-(\gamma / 2)^{2}=-(\bar{\alpha}+\gamma / 2)^{2},
$$

where $r \geq 0$ and $-\pi<\theta \leq \pi$. Then, the solution of the characteristic function is obtained as

$$
\bar{\alpha}=-\frac{\gamma}{2} \pm \mathrm{i} \sqrt{r} e^{\mathrm{i} \theta / 2}
$$

This yields the oscillating solution of $a_{\mu}(t)$ as

$$
\bar{a}_{\mu}(t)=\bar{a}_{\mu}(0) \exp \left[-\frac{\gamma}{2} t \pm \mathrm{i}\left(\sqrt{r} e^{\mathrm{i} \theta / 2}\right) t\right] .
$$

Since

$$
\sqrt{r} e^{\mathrm{i} \theta / 2}=\sqrt{r} \cos \left(\frac{\theta}{2}\right)+\mathrm{i} \sqrt{r} \sin \left(\frac{\theta}{2}\right)
$$

we have

$$
\bar{a}_{\mu}(t)=\bar{a}_{\mu}(0) \exp \left[-\left(\frac{\gamma}{2} \pm \sqrt{r} \sin \left(\frac{\theta}{2}\right)\right) t\right.
$$

$$
\left.\pm \mathrm{i} \sqrt{r} \cos \left(\frac{\theta}{2}\right) t\right]
$$

From the structure of (47), we can recognize that $\bar{a}_{\mu}(t)$ diverges exponentially if

$$
\sqrt{r}\left|\sin \left(\frac{\theta}{2}\right)\right|>\frac{\gamma}{2}
$$

This phenomena can be considered as the flaming prevalent on social networks [20].

In order to prevent and/or suppress flaming, an effective control strategy is to make the networks symmetrizable. This approach makes $\mathcal{L}_{\mathrm{I}}=\mathbf{O}$ based on the decomposition (36). At this time, the diversity of the decomposition (36) leads to control flexibility. More details are discussed in Sect. 8. An actual solution has been investigated [21].

\section{Fundamental Equation of Oscillation on Networks}

In this section, based on the decomposition (39), we consider a theoretical framework that ties the existence of $S_{\mathrm{I}}$ to the behavior of oscillation dynamics. That is, we consider a model that describes how independent oscillation modes under the condition $\boldsymbol{S}_{\mathrm{I}}=\mathbf{O}$ change when $\boldsymbol{S}_{\mathrm{I}} \neq \mathbf{O}$, and clarifies how different oscillation modes are coupled. This consideration is a significant breakthrough in applying the spectral graph theory to directed graphs.

In order to understand how $S_{\mathrm{I}}$ triggers coupling between different oscillation modes, systematically, we describe the effect of $S_{\text {I }}$ on the oscillation dynamics based on the eigenbasis of $\boldsymbol{S}_{0}$. This is because the eigenbasis of $\boldsymbol{S}_{0}$ describes independent oscillation modes under $\boldsymbol{S}_{\mathrm{I}}=\mathbf{O}$.

Let the eigenvalues of $\boldsymbol{S}_{0}$ be $\lambda_{\mu}(\mu=0,1, \ldots, n-1)$ as per (12), and eigenvector $\boldsymbol{v}_{\mu}$ associated with $\lambda_{\mu}$ be chosen as per (13). In addition, let the diagonalized matrix of $\boldsymbol{S}_{0}$ by the orthogonal matrix $\boldsymbol{P}:=\left(\boldsymbol{v}_{0}, \boldsymbol{v}_{1}, \ldots, \boldsymbol{v}_{n-1}\right)$ be

$$
\boldsymbol{\Lambda}_{0}:={ }^{t} \boldsymbol{P} \boldsymbol{S}_{0} \boldsymbol{P},
$$

where $\boldsymbol{\Lambda}_{0}=\operatorname{diag}\left(\lambda_{0}, \ldots, \lambda_{n-1}\right)$.

By using $\boldsymbol{\psi}(t):={ }^{t} \boldsymbol{P} \boldsymbol{y}$ and $\boldsymbol{\Lambda}_{\mathrm{I}}:={ }^{t} \boldsymbol{P} \boldsymbol{S}_{\mathrm{I}} \boldsymbol{P}$, the equation of motion (41) can be rewritten as

$$
\frac{\mathrm{d}^{2} \psi(t)}{\mathrm{d} t^{2}}=-\left(\boldsymbol{\Lambda}_{0}+\Lambda_{\mathrm{I}}\right) \psi(t)
$$

If $\boldsymbol{S}_{\mathrm{I}}=\boldsymbol{\Lambda}_{\mathrm{I}}=\mathbf{O}$ (i.e. symmetrizable graph), the solution of (48) is obtained as

$$
\psi(t)=\exp \left( \pm \mathrm{i} \Lambda_{0}^{1 / 2} t\right) \psi(0) .
$$

This means that the oscillation dynamics on networks can be decomposed into $n$ independent harmonic oscillators (eigenfrequencies $\omega_{\mu}=\sqrt{\lambda_{\mu}}$ ). Since the decomposition into independent oscillation modes fails if $\boldsymbol{\Lambda}_{\mathrm{I}} \neq \mathbf{O}$, it is necessary to evaluate the effect of $\boldsymbol{\Lambda}_{\mathrm{I}}$ appropriately.

In order to evaluate the effect of $\boldsymbol{\Lambda}_{\mathrm{I}}$ causally, the solution $\psi(t)$ of the equation of motion (48) should be given by a product-form solution of effects from $\boldsymbol{\Lambda}_{0}$ and $\boldsymbol{\Lambda}_{\mathrm{I}}$ [22]. 
As the first trial, let us consider a simple product-form solution. Let us consider the following equations:

$$
\begin{aligned}
\frac{\mathrm{d}^{2} \boldsymbol{\phi}_{0}(t)}{\mathrm{d} t^{2}} & =-\boldsymbol{\Lambda}_{0} \boldsymbol{\phi}_{0}(t), \\
\frac{\mathrm{d}^{2} \boldsymbol{\phi}_{\mathrm{I}}(t)}{\mathrm{d} t^{2}} & =-\left(\boldsymbol{\Phi}_{0}(-t) \boldsymbol{\Lambda}_{\mathrm{I}} \boldsymbol{\Phi}_{0}(t)\right) \boldsymbol{\phi}_{\mathrm{I}}(t),
\end{aligned}
$$

where $\phi_{0}(t)$ and $\phi_{\mathrm{I}}(t)$ are the solutions of these equation, respectively. In addition, we define diagonal matrix $\boldsymbol{\Phi}_{0}(t)$ whose diagonal components are the components of the vector $\boldsymbol{\phi}_{0}(t)$, that is, for ${ }^{t} \boldsymbol{\phi}_{0}(t)=\left(\phi_{0}(1 ; t), \ldots, \phi_{0}(n ; t)\right)$,

$$
\boldsymbol{\Phi}_{0}(t)=\left(\begin{array}{cccc}
\phi_{0}(1 ; t) & 0 & \ldots & 0 \\
0 & \phi_{0}(2 ; t) & \vdots & \vdots \\
\vdots & \ldots & \ddots & \vdots \\
0 & 0 & 0 & \phi_{0}(n ; t)
\end{array}\right) .
$$

Then, we assume that the solution, $\psi(t)$, is given in productform as

$$
\boldsymbol{\psi}(t)=\boldsymbol{\Phi}_{0}(t) \phi_{\mathrm{I}}(t) .
$$

That is, for ${ }^{t} \phi_{\mathrm{I}}(t)=\left(\phi_{\mathrm{I}}(1 ; t), \ldots, \phi_{\mathrm{I}}(n ; t)\right)$,

$$
\psi(t)=\boldsymbol{\Phi}_{0}(t) \phi_{\mathrm{I}}(t)=\left(\begin{array}{c}
\phi_{0}(1 ; t) \phi_{\mathrm{I}}(1 ; t) \\
\phi_{0}(2 ; t) \phi_{\mathrm{I}}(2 ; t) \\
\vdots \\
\phi_{0}(n ; t) \phi_{\mathrm{I}}(n ; t)
\end{array}\right) .
$$

By substituting (52) into the equation of motion (48), we have

$$
\begin{aligned}
\frac{\mathrm{d}^{2} \boldsymbol{\psi}(t)}{\mathrm{d} t^{2}} & =-\frac{\mathrm{d}^{2}}{\mathrm{~d} t^{2}}\left(\boldsymbol{\Phi}_{0}(t) \boldsymbol{\phi}_{\mathrm{I}}(t)\right) \\
& =-\left(\boldsymbol{\Lambda}_{0}+\boldsymbol{\Lambda}_{\mathrm{I}}\right) \boldsymbol{\psi}(t)+2 \frac{\mathrm{d} \boldsymbol{\Phi}_{0}(t)}{\mathrm{d} t} \frac{\mathrm{d} \boldsymbol{\phi}_{\mathrm{I}}(t)}{\mathrm{d} t} \\
& \neq-\left(\boldsymbol{\Lambda}_{0}+\boldsymbol{\Lambda}_{\mathrm{I}}\right) \boldsymbol{\psi}(t) .
\end{aligned}
$$

This means the trial to make a product-form solution (52) does not succeed.

The reason for this failure of the product-form solution is the existence of the cross term in (53). Moreover, the existence of the cross term is caused by the fact that (48) is a second-order differential equation. So, to obtain a firstorder differential equation, we consider a linear operator $\boldsymbol{H}$ that satisfies

$$
\boldsymbol{H}^{2}=\boldsymbol{\Lambda}_{0}+\boldsymbol{\Lambda}_{\mathrm{I}}
$$

By choosing $\boldsymbol{H}$ appropriately, we propose the fundamental equation of oscillation dynamics on networks [22] as

$$
\mathrm{i} \frac{\mathrm{d} \boldsymbol{\psi}(t)}{\mathrm{d} t}=\boldsymbol{H} \boldsymbol{\psi}(t) .
$$

Note that this equation is formally equivalent to the Schrödinger equation (in a broad sense, see Sect. 8) in quantum theory (physical constant $\hbar$ is ignored). Since the solution of (55) satisfies

$$
\frac{\mathrm{d}^{2} \boldsymbol{\psi}(t)}{\mathrm{d} t^{2}}=-\mathrm{i} \boldsymbol{H} \frac{\mathrm{d} \boldsymbol{\psi}(t)}{\mathrm{d} t}=-\boldsymbol{H}^{2} \boldsymbol{\psi}(t)=-\left(\boldsymbol{\Lambda}_{0}+\boldsymbol{\Lambda}_{\mathrm{I}}\right) \boldsymbol{\psi}(t),
$$

the solution of (55) is the solution of the original equation (48).

Next, we consider solution $\psi(t)$ in product-form. By using $\boldsymbol{H}_{0}:=\boldsymbol{\Lambda}_{0}^{1 / 2}$, the decomposition of $\boldsymbol{H}$ is given as

$$
\boldsymbol{H}=\boldsymbol{H}_{0}+\boldsymbol{H}_{\mathrm{I}} .
$$

We assume that solution $\psi(t)$ is given in product-form as

$$
\psi(t)=\Psi_{0}(t) \psi_{\mathrm{I}}(t),
$$

where the functions appearing in (58) are given by the vectors $\psi_{0}(t)$ and $\psi_{\mathrm{I}}(t)$ that satisfy

$$
\begin{aligned}
\mathrm{i} \frac{\mathrm{d}}{\mathrm{d} t} \boldsymbol{\psi}_{0}(t) & =\boldsymbol{H}_{0} \boldsymbol{\psi}_{0}(t), \\
\mathrm{i} \frac{\mathrm{d}}{\mathrm{d} t} \psi_{\mathrm{I}}(t) & =\left(\boldsymbol{\Psi}_{0}(-t) \boldsymbol{H}_{\mathrm{I}} \boldsymbol{\Psi}_{0}(t)\right) \boldsymbol{\psi}_{\mathrm{I}}(t)
\end{aligned}
$$

and the diagonal matrix $\boldsymbol{\Psi}_{0}(t)$ whose diagonal components are the components of $\psi_{0}(t)$.

Substituting (58) into the fundamental equation (55) yields

$$
\begin{aligned}
\mathrm{i} \frac{\mathrm{d} \psi(t)}{\mathrm{d} t} & =\mathrm{i} \frac{\mathrm{d}}{\mathrm{d} t}\left(\boldsymbol{\Psi}_{0}(t) \boldsymbol{\psi}_{\mathrm{I}}(t)\right) \\
& =\boldsymbol{H}_{0} \boldsymbol{\Psi}_{0}(t) \boldsymbol{\psi}_{\mathrm{I}}(t)+\boldsymbol{\Psi}_{0}(t)\left(\boldsymbol{\Psi}_{0}(-t) \boldsymbol{H}_{\mathrm{I}} \boldsymbol{\Psi}_{0}(t)\right) \boldsymbol{\psi}_{\mathrm{I}}(t) \\
& =\left(\boldsymbol{H}_{0}+\boldsymbol{H}_{\mathrm{I}}\right) \boldsymbol{\psi}(t)=\boldsymbol{H} \boldsymbol{\psi}(t) .
\end{aligned}
$$

This means that the trial, attempting to make a product-form solution, (58) has succeeded.

The representations of (55) is called the Scrödinger picture, while that of (60) is called the interaction picture (or the Dirac picture) (see Sect. 8).

Using (58)-(60), for the initial condition $\boldsymbol{\Psi}_{0}\left(t_{0}\right)=\boldsymbol{I}$ at $t=t_{0}$, the solution $\Psi_{0}(t)$ is obtained from (59), as

$$
\boldsymbol{\Psi}_{0}(t)=\exp \left(-\mathrm{i} \boldsymbol{H}_{0}\left(t-t_{0}\right)\right) \text {. }
$$

So, the solution $\psi(t)$ of (55) is obtained as

$$
\boldsymbol{\psi}(t)=\exp \left(-\mathrm{i} \boldsymbol{H}_{0}\left(t-t_{0}\right)\right) \boldsymbol{\psi}_{\mathrm{I}}(t) .
$$

For analyzing this solution, we can apply the perturbation theory developed and used in quantum theory, which suggests that the effect of $\mathcal{L}_{\mathrm{I}}$ can be understood causally.

Although method of determining $\boldsymbol{H}$ from (54) is not unique, there is a degree of freedom that permits the more convenient choice to made. With regard to this issue, a simple method based on the algebraic structure of the special unitary group $\mathrm{SU}(2)$ has been investigated [22].

\section{8. $\quad$ Related Work}

Almost all research on the dynamics on networks based on the spectral graph theory focuses on the diffusion process, 
Markov chain, or random walk on networks. For example, if $k_{i j}$ of the symmetric Laplacian matrix $\boldsymbol{L}$ shown in Sect. 2.2 is taken to be the diffusion coefficient of the link between nodes $i$ and $j$, the diffusion equation on networks can be written as

$$
\frac{\mathrm{d} \boldsymbol{x}(t)}{\mathrm{d} t}=-\boldsymbol{L} \boldsymbol{x}(t)
$$

As another example, if $w_{i j}$ of the asymmetric Laplacian matrix $\mathcal{L}$ is taken to be the transition rate from state $i$ to state $j$, the temporal evolution equation describing the corresponding continuous-time Markov chain can be written as

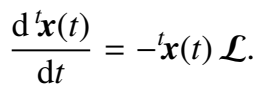

A comparison to the equation of motion for the oscillation on networks (15) or (40), shows that (63) and (64) are the first-order differential equations with respect to time, while (15) and (40) are second-order equations.

The condition (5) means Newton's third law in the oscillation model, but another interpretation is also possible. This condition is known as the detailed balance condition or the local balance equation, and this guarantees the existence of the equilibrium solution of the corresponding Markov chain. In addition, the Markov chain that satisfies the condition (5) is called the reversible Markov chain.

Next, let us consider the discrete-time Markov chain. The probability transition matrix $\mathcal{P}=\left[p_{i j}\right]$ is defined as

$$
\mathcal{P}:=\mathcal{D}^{-1} \mathcal{A} \text {. }
$$

Here, $p_{i j}$ denotes the transition probability from state $i$ to state $j$ in one unit of time, and the row sum of $\mathcal{P}$ is $\sum_{j=1}^{n} p_{i j}=1$. Then, the relationship between two states at discrete time $t_{k}$ and at $t_{k+1}$ is given as

$$
{ }^{t} \boldsymbol{x}\left(t_{k+1}\right)={ }^{t} \boldsymbol{x}\left(t_{k}\right) \mathcal{P} \text {. }
$$

Rewriting this using the Laplacian matrix yields

$$
{ }^{t} \boldsymbol{x}\left(t_{k+1}\right)-{ }^{t} \boldsymbol{x}\left(t_{k}\right)=-{ }^{t} \boldsymbol{x}\left(t_{k}\right)(\boldsymbol{I}-\mathcal{P})=-{ }^{t} \boldsymbol{x}\left(t_{k}\right)\left(\mathcal{D}^{-1} \mathcal{L}\right) .
$$

As in this example, in order to make the row sum of transition probability matrix equal 1 in the discrete-time Markov chain, the Laplacian matrix is often normalized by using the node degree, and

$$
\mathcal{N}:=\mathcal{D}^{-1 / 2} \mathcal{L} \mathcal{D}^{-1 / 2}
$$

is called the normalized Laplacian matrix.

Let us consider that we apply a scaling procedure similar to that in Sect. 2.2 for the scaled Laplacian matrix to the matrix $\left(\mathcal{D}^{-1} \mathcal{L}\right)$ (asymmetric matrix, in general) of (65). If ${ }^{t} \boldsymbol{m}=\left(m_{1}, \ldots, m_{n}\right)$ is the left eigenvector associated with the eigenvalue 0 , that is

$$
{ }^{t} \boldsymbol{m}\left(\mathcal{D}^{-1} \mathcal{L}\right)={ }^{t} \mathbf{0}
$$

the (normalized) the digraph Laplacian matrix (or called
Diplacian [24]) $\boldsymbol{\Gamma}$ is defined as

$$
\boldsymbol{\Gamma}:=\boldsymbol{M}^{+1 / 2}\left(\mathcal{D}^{-1} \mathcal{L}\right) \boldsymbol{M}^{-1 / 2},
$$

where $\boldsymbol{M}=\operatorname{diag}\left(m_{1}, \ldots, m_{n}\right)$. Similarly, for the Laplacian matrix $\mathcal{L}$ of a directed graph, $\boldsymbol{\Gamma}$ is defined as

$$
\Gamma:=\boldsymbol{M}^{+1 / 2} \mathcal{L} \boldsymbol{M}^{-1 / 2},
$$

where $\boldsymbol{M}=\operatorname{diag}\left(m_{1}, \ldots, m_{n}\right)$ and ${ }^{t} \boldsymbol{m}=\left(m_{1}, \ldots, m_{n}\right)$ satisfies (4).

Here, we discuss the difference between the scaled Laplacian matrix $S$ in (38) and the Diplacian $\boldsymbol{\Gamma}$ in (66). The difference is in the scaling matrices $\boldsymbol{M}_{0}$ and $\boldsymbol{M}$. In the Diplacian, $m_{i}$ of $\boldsymbol{M}$ satisfies (4). On the other hand, in the scaled Laplacian matrix, $m_{i}$ of $\boldsymbol{M}_{0}$ is chosen by using (37), where $\mathcal{L}_{0}$ is a symmetrizable Laplacian matrix included in $\mathcal{L}$, and $\boldsymbol{S}$ is defined using $\boldsymbol{M}_{0}$ as (38). Thus $\boldsymbol{S}$ and $\boldsymbol{\Gamma}$ are, in general, different, but $\boldsymbol{S}=\boldsymbol{\Gamma}$ when the Laplacian matrix $\mathcal{L}$ is symmetrizable.

Note that the Diplacian is uniquely determined for a given directed graph. The decomposition of the Diplacian is discussed in [24] as,

$$
\boldsymbol{\Gamma}=\frac{\boldsymbol{\Gamma}+{ }^{t} \boldsymbol{\Gamma}}{2}+\frac{\boldsymbol{\Gamma}-{ }^{t} \boldsymbol{\Gamma}}{2} .
$$

If $\boldsymbol{\Gamma}$ is a symmetric matrix (directed graph is symmetrizable), the second term of the right hand side vanishes. Therefore, the second term denotes a certain degree of asymmetry. The decomposition (67) is also unique for a given directed graph.

On the other hand, the scaled Laplacian matrix $S$ depends on the choice of the symmetrizable Laplacian matrix $\mathcal{L}_{0}$, that is, it depends on the decomposition (36). For example, Fig. 12 shows three decompositions into a symmetrizable graph and a one-way link graph. Note that, for a symmetrizable graph, the products of link weights around each closed loop are the same in both directions. The strength of the degree of freedom of the decomposition lies in the flexibility of choosing which link should be controlled to reduce flaming. By controlling one-way links in any of possible decomposition, we can make the directed graph symmetrizable. In addition, selecting an appropriate symmetrizable graph means selecting an appropriate basis (the eigenbasis of $\boldsymbol{S}_{0}$ ) of an $n$-dimensional state space to more simply describe the dynamics on the directed graph.

To highlight this strength, let us compare the decomposition with the Diplacian. The Diplacian for the directed graph shown in Fig. 12 is uniquely obtained as

$$
\boldsymbol{\Gamma}=\left(\begin{array}{cccc}
5 & -\frac{\sqrt{62}}{5} & -3 \sqrt{\frac{93}{55}} & 0 \\
-20 \sqrt{\frac{2}{31}} & 5 & -3 \sqrt{\frac{30}{11}} & -15 \sqrt{\frac{6}{71}} \\
-\sqrt{\frac{55}{93}} & -\sqrt{\frac{11}{30}} & 7 & -2 \sqrt{\frac{55}{71}} \\
0 & -\frac{1}{5} \sqrt{\frac{142}{3}} & -\sqrt{\frac{71}{55}} & 5
\end{array}\right) .
$$

Therefore, the anti-symmetric graph given by the decomposition (67) includes all the links in the original directed 


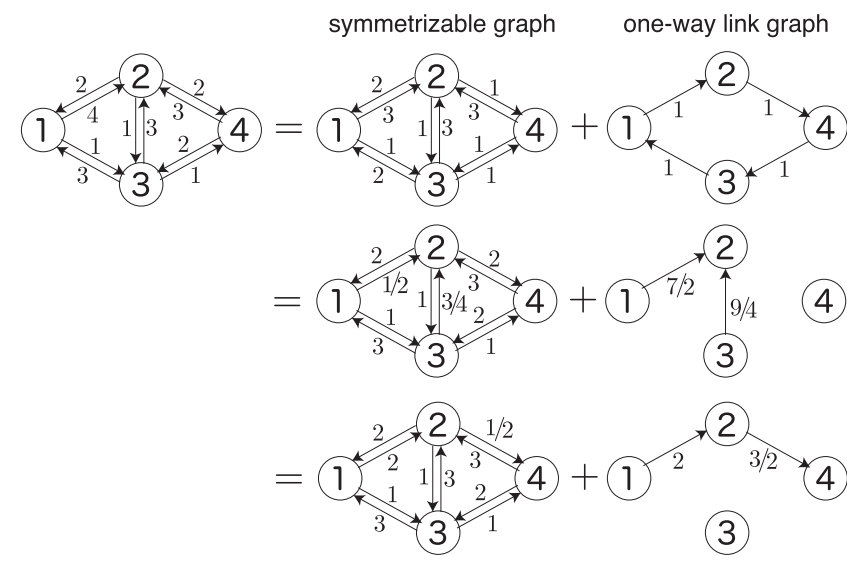

Fig. 12 Examples of decompositions of a directed graph into symmetrizable and one-way link graphs.

graph. This is too complicated to control corresponding links.

Next, we discuss the relationship between our oscillation model and the quantum walk, in particular, the continuous-time quantum walk (CTQW) [27]. First, we briefly summarize some equations that appear in quantum theory. To simplify the discussion, we adopt the natural unit system, that is, the speed of light $c=1$ and the reduced Planck constant $\hbar=1$.

The temporal evolution equation in quantum theory consists of the state vector and the linear operator that acts the state vector. Depending on whether the time dependency lies in the state vector or the linear operator, there are typically two (precisely three as is discussed later) different representations of the temporal evolution equation. The formulation in which the state vector is time dependent is called the Schrödinger picture, whereas that in which the linear operator is time dependent is called the Heisenberg picture. Here, we adopt the Schrödinger picture; its temporal evolution equation is

$$
\mathrm{i} \frac{\partial}{\partial t} \psi(\boldsymbol{x}, t)=\hat{\boldsymbol{H}} \psi(\boldsymbol{x}, t)
$$

where $\psi(\boldsymbol{x}, t)$ is a wave function that describes the system state and $\hat{\boldsymbol{H}}$ is a linear operator (called the Hamiltonian in quantum theory) that generates temporal evolution. This equation is called Schrödinger equation (in a broad sense).

In quantum theory, a different temporal evolution equation appears depending on whether special relativity is satisfied or not. In non-relativistic quantum theory, the temporal evolution equation (wave equation) for a free particle of mass $m$ is written as

$$
\mathrm{i} \frac{\partial}{\partial t} \psi(\boldsymbol{x}, t)=\frac{-1}{2 m} \nabla^{2} \psi(\boldsymbol{x}, t),
$$

and this is called the Schrödinger equation. On the other hand, in relativistic quantum theory, the wave equation for a free particle of mass $m$ is written as

$$
\frac{\partial^{2}}{\partial t^{2}} \psi(\boldsymbol{x}, t)=\left(\nabla^{2}-m^{2}\right) \psi(\boldsymbol{x}, t)
$$

and this is called the Klein-Gordon equation [25]. In order to derive a first-order differential equation with respect to time from the Klein-Gordon equation, by choosing some quantities for $\alpha, \beta$ that satisfy $(-\mathrm{i} \alpha \cdot \nabla+\beta m)^{2}=-\nabla^{2}+m^{2}$, we have

$$
\mathrm{i} \frac{\partial}{\partial t} \psi(\boldsymbol{x}, t)=(-\mathrm{i} \boldsymbol{\alpha} \cdot \nabla+\beta m) \psi(\boldsymbol{x}, t) .
$$

This is the Dirac equation [25]. In particular, the Dirac equation for massless particle $m=0$ is called the Weyl equation.

Since the Laplacian matrix gives the second order difference on the network, we can recognize that (40) and (48) correspond to the Klein-Gordon equation for massless particles, and (55) corresponds to the Weyl equation (or the Dirac equation for massless particle).

The representation of (55) corresponds to the Scrödinger picture, while that of (60) corresponds to the interaction picture (or the Dirac picture) of quantum theory. The interaction picture is an intermediate picture between the Schrödinger and Heisenberg pictures, that is, both the state vector and linear operator have time dependency. In addition, (60) is called the Tomonaga-Schwinger equation. The temporal evolution equation (60) in the interaction picture is convenient for calculating perturbation that is a key element of quantum field theory. Since the relativistic quantum theory is closely related to the quantum field theory [26], our oscillation model, described by (60), is expected to introduce the quantum field theory to network analysis.

On the other hand, CTQW is a generalization of the Markov chain by introducing an imaginary unit to (64), the temporal evolution equation is given by

$$
\frac{\mathrm{d}^{t} \boldsymbol{x}(t)}{\mathrm{d} t}=-\mathrm{i}^{t} \boldsymbol{x}(t) \mathcal{L}
$$

This equation corresponds to the Schrödinger equation for non-relativistic quantum theory.

It goes without saying that it differs in that the object being considered by the oscillation model on networks and the random walk on networks, but the key difference is in the relation between the Hamiltonian and the Laplacian matrix. In CTQW, the generator of temporal evolution (the Hamiltonian in quantum theory) directly corresponds to the Laplacian matrix, whereas in our oscillation model, the square of the Hamiltonian corresponds to the Laplacian matrix.

\section{Conclusions}

This paper has proposed an oscillation model on networks for analyzing that complex dynamics of activity on social media networks, and shown the significance of and progress in four research topics derived from the proposed oscillation model.

First of all, we have categorized link asymmetries into two types: symmetrizable and unsymmetrizable. For symmetrizable directed graphs, the oscillation dynamics can be analyzed by using the symmetric scaled Laplacian matrix. The significance of the oscillation model on symmetrizable 
directed graphs is the relationship between the oscillation energy of each node and node centrality. The oscillation energy gives a generalized notion of the conventional node centrality (the degree and between centralities). Currently, various generalizations of node centralities are under investigation [16], [17].

Conversely, as a method for determining the structure of the Laplacian matrix from observations of generalized node centrality, the network resonance method has been proposed. This method enables us to estimate information about eigenvalues and eigenvectors of the scaled Laplacian matrix based on resonance. Currently, we aim to establish a technique to reproduce the Laplacian matrix of social media networks by applying the network resonance method [19], [28], if this is realized, the strength of the effective influence among users can be estimated.

Next, we have generalized the oscillation model for application to general directed graphs including unsymmetrizable graphs. In this case, eigenvalues might become complex numbers, and we have shown that the oscillation energy diverges in a certain condition. This model can be considered as a dynamical model of the flaming phenomenon on social media networks. Currently, based on this model, countermeasures to are being investigated [21].

Finally, we have proposed the fundamental equation of oscillation dynamics on a general directed graphs. In order to understand the behavior of unsymmetrizable graphs, the solution of the oscillation should be given in productform that involves the effects of both the symmetrizable part and the other parts of the graphs. As a result of postulating the product-form solution, we derived a fundamental equation that mirrors a quantum theoretical equation. Therefore, it can be recognized that quantization is more than a specialized procedure for describing the physical behavior of electrons, photons, and other small particles, in that it can be seen as a framework for understanding the causality of oscillation dynamics.

On the other hand, there are two the essential differences between quantum theory and the proposed oscillation model. The first is the degree of freedom. Although quantum field theory has an infinite degree of freedom, the degree of freedom of the oscillation model is $n$, that is the number of nodes. The second is the characteristics of the linear operators. The linear operators corresponding to physical quantity appearing in quantum theory are Hermitian, and always have real-valued eigenvalues. This is a major premise used in describing the physical world. On the other hand, for the proposed oscillation model, $\boldsymbol{S}$ and $\boldsymbol{H}$ for a general directed graph are not, in general, Hermitian. This causes the flaming phenomenon which violates the energy conservation law.

The fundamental equation (55) or (60) provides a significant breakthrough in establishing spectral graph theory for directed graphs; this is our current research topic [22], [23].

\section{Acknowledgments}

The authors would like to thank Mr. Satoshi Furutani of Tokyo Metropolitan University for his help with the numerical experiments. This research is supported by a Grant-in-Aid for Scientific Research (B) No. 26280032 (2014-2016) and No. 17H01737 (2017-2019) from the Japan Society for the Promotion of Science.

\section{References}

[1] S. Wasserman and K. Faust, Social Network Analysis: Methods and Applications, Cambridge University Press, 1994.

[2] P.J. Carrington, J. Scott, and S. Wasserman, Models and Methods in Social Network Analysis, Cambridge University Press, 2005.

[3] A. Mislove, M. Marcon, K.P. Gummadi, P. Druschel and B. Bhattacharjee, "Measurement and analysis of online social networks," Proc. ACM SIGCOMM Conference on Internet Measurement, pp.29-42, 2007.

[4] F.R.K. Chung, "Lectures on spectral graph theory," CBMS Lecture Notes, AMS Publications, Providence, 1995.

[5] D. Spielman, "Spectral graph theory," in Combinatorial Scientific Computing, U. Naumann and O. Schenk, eds., Chapter 18, pp.495524, Chapman and Hall/CRC, 2012.

[6] M.E.J. Newman, "The graph Laplacian," in Networks: An Introduction, Section 6.13, pp.152-157, Oxford University Press, 2010.

[7] A. Jadbabaie, J. Lin, and A.S. Morse, "Coordination of groups of mobile autonomous agents using nearest neighbor rules," IEEE Trans. Autom. Control, vol.48, no.6, pp.988-1001, 2003,

[8] R. Olfati-Saber and R.M. Murray, "Consensus problems in networks of agents with switching topology and time-delays," IEEE Trans. Autom. Control, vol.49, no.9, pp.1520-1533, 2004.

[9] D.K. Hammond, P. Vandergheynst, and R. Gribonval, "Wavelets on graphs via spectral graph theory," Appl. Comput. Harmon. Anal., vol.30, no.2, pp.129-150, 2011.

[10] D.I. Shuman, S.K. Narang, P. Frossard, A. Ortega, and P. Vandergheynst, "The emerging field of signal processing on graphs: Extending high-dimensional data analysis to networks and other irregular domains," IEEE Signal Process. Mag., vol.30, no.3, pp.83-98, 2013.

[11] A. Sandryhaila and J.M.F. Moura, "Discrete signal processing on graphs,” IEEE Trans. Signal Process., vol.61, no.7, pp.1644-1656, 2013.

[12] A. Sandryhaila and J.M.F. Moura, "Big data analysis with signal processing on graphs: Representation and processing of massive data sets with irregular structure," IEEE Signal Process. Mag., vol.31, no.5, pp.80-90, 2014.

[13] Y. Kuramoto, Chemical Oscillations, Waves, and Turbulence, Dover Books on Chemistry, 2003.

[14] M. Aida, C. Takano, and M. Murata, "Oscillation model for network dynamics caused by asymmetric node interaction based on the symmetric scaled Laplacian matrix," The 12th International Conference on Foundations of Computer Science (FCS 2016), July 2016.

[15] M, Aida, "Oscillation model for describing propagation of activities on network caused by asymmetric node interactions," Keynote Speech, IEICE Information and Communication Technology Forum 2016 (ICTF 2016), July 2016.

[16] C. Takano and M. Aida, "Proposal of new index for describing node centralities based on oscillation dynamics on networks," IEEE GLOBECOM 2016, Dec. 2016.

[17] C. Takano and M. Aida, "Fundamental framework for describing various node centralities using an oscillation model on social media networks," IEEE ICC 2017, May 2017.

[18] S. Furutani, C. Takano and M. Aida, "Proposal of the network res- 
onance method for estimating eigenvalues of the scaled Laplacian matrix," The 8th International Conference on Intelligent Networking and Collaborative Systems (INCoS 2016) Workshop (WIND 2016), Sept. 2016

[19] S. Furutani, C. Takano and M. Aida, "Method for estimating the eigenvectors of a scaled Laplacian matrix using the resonance of oscillation dynamics on networks," IEEE/ACM ASONAM 2017, pp.615-618, Aug. 2017.

[20] M. Aida, C. Takano and M. Murata, "Dynamical model of flaming phenomena in on-line social networks," IEEE/ACM IASONAM 2017, pp.1164-1171, Aug. 2017.

[21] C. Takano and M. Aida, "Countermeasure technology for flaming on social media networks based on the oscillation model," IEICE General Conf., BS-3-3, March 2017(in Japanese).

[22] M. Aida, "Modelling and applications of oscillation dynamics on networks generated by asymmetric interaction between nodes," IEICE Technical Report, CCS2015-66, March 2016 (in Japanese).

[23] M. Aida, "Fundamental equation of oscillation dynamics on networks," IEICE General Conf., BS-3-4, March 2017 (in Japanese).

[24] Y. Li and Z.-L. Zhang, "Digraph Laplacian and the degree of asymmetry," Internet Mathematics, vol.8, no.4, pp.381-401, Dec. 2012.

[25] J.D. Bjorken and S.D. Drell, Relativistic Quantum Mechanics, MacGraw-Hill, New York, 1964.

[26] J.D. Bjorken and S.D. Drell, Relativistic Quantum Fields, MacGraw-Hill, New York, 1965.

[27] N. Konno, "Quantum walks," in Quantum Potential Theory, Lecture Note in Mathematics, vol.1954, pp.309-452, Springer-Verlag, Heidelberg, Oct. 2008.

[28] S. Sugimoto and M. Aida, "Estimating the structure of social networks from incomplete set of observed information by using compressed sensing," IEEE LATINCOM 2017, Nov. 2017.

\section{Appendix: Canonical Equation of Motion Non-Damped Oscillation Model on Networks}

We use Hamilton's canonical formalism to derive the equation of motion for the non-damped oscillation model shown in Sect. 3.1.

Let the spring constant for the link between node $i$ and node $j$ be the link weight $k_{i j}>0$. In addition, we assign mass $m_{i}>0$ to each node $i$. Let $x_{i}=x_{i}(t)$ be the displacement of node $i$ and $p_{i}=p_{i}(t)$ be its conjugate momentum. Then, Hamiltonian $\mathcal{H}$ of our coupled oscillator system is expressed as

$$
\mathcal{H}:=\sum_{i \in V} \frac{\left(p_{i}\right)^{2}}{2 m_{i}}+\sum_{(i, j) \in E} \frac{k_{i j}}{2}\left(x_{i}(t)-x_{j}(t)\right)^{2} .
$$

By following the canonical formalism, the canonical equations of motion are obtained as

$$
\begin{aligned}
\frac{\mathrm{d} p_{i}(t)}{\mathrm{d} t} & =-\frac{\partial \mathcal{H}}{\partial x_{i}}=-\sum_{j \in V} L_{i j} x_{j}(t), \\
\frac{\mathrm{d} x_{i}(t)}{\mathrm{d} t} & =\frac{\partial \mathcal{H}}{\partial p_{i}}=\frac{p_{i}}{m_{i}},
\end{aligned}
$$

and by eliminating $p_{i}$ from the equations of motion, we have the following wave equation as the equation of motion:

$$
\boldsymbol{M} \frac{\mathrm{d}^{2} \boldsymbol{x}(t)}{\mathrm{d} t^{2}}=-\boldsymbol{L} \boldsymbol{x}(t),
$$

where $\boldsymbol{x}(t)$ is the node displacement vector

$$
\boldsymbol{x}(t):={ }^{t}\left(x_{1}(t), \ldots, x_{n}(t)\right),
$$

and $\boldsymbol{M}$ is the mass matrix $\boldsymbol{M}:=\operatorname{diag}\left(m_{1}, \ldots, m_{n}\right)$.

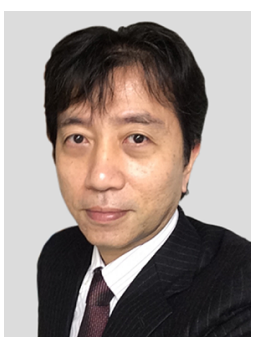

Masaki Aida received his B.S. degree in Physics and M.S. degree in Atomic Physics from St. Paul's University, Tokyo, Japan, in 1987 and 1989, respectively, and his Ph.D. in Telecommunications Engineering from the University of Tokyo, Japan, in 1999. In April 1989, he joined NTT Laboratories. From April 2005 to March 2007, he was an Associate Professor at the Faculty of System Design, Tokyo Metropoli$\tan$ University. He has been a Professor of the Graduate School of Systems Design, Tokyo Metropolitan University since April 2007. His current interests include analysis of social network dynamics and distributed control of computer communication networks. He received the Best Tutorial Paper Award and the Best Paper Award of IEICE Communications Society in 2013 and 2016, respectively. He is a member of the IEEE and the Operations Research Society of Japan.

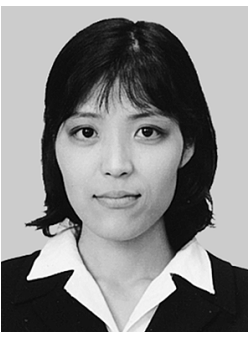

Chisa Takano received a B.E. degree in Telecommunication Engineering from Osaka University, Japan, in 2000, and a Ph.D. in System Design Engineering from Tokyo Metropolitan University, Japan, in 2008. In 2000, she joined the Traffic Research Center, NTT Advanced Technology Corporation. Since April 2008, she has been an Associate Professor at the Graduate School of Information Sciences, Hiroshima City University. Her research interests are computer networks and distributed systems. She received the IEICE Young Researchers'Award in 2003. She received the Information Network Research Awards from the IEICE in 2004, 2012, and 2015. She is a member of IEEE (U.S.A.).

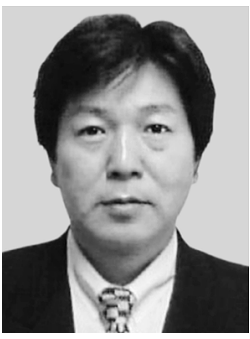

Masayuki Murata received the M.E. and D.E. degrees in Information and Computer Sciences from Osaka University, Japan, in 1984 and 1988, respectively. In April 1984, he joined IBM Japan's Tokyo Research Laboratory, as a Researcher. From September 1987 to January 1989, he was an Assistant Professor with the Computation Center, Osaka University. In February 1989, he moved to the Department of Information and Computer Sciences, Faculty of Engineering Science, Osaka University. In April 1999, he became a Professor of Osaka University, and since April 2004 he has been with the Graduate School of Information Science and Technology, Osaka University. He has contributed more than four hundred and fifty papers to international and domestic journals and conferences. His research interests include computer communication networks and performance modeling and evaluation. He is a member of the IEEE, ACM, IEICE, the Internet Society, and IPSJ. 